\title{
Differential Diagnosis
}

\section{Osteochondrosis Mimicking Spondylitis}

- A 59-year-old woman

- Patient with chronic back pain

- No increase of the inflammatory markers

Fig. 1 Sagittal T1-weighted image (a), sagittal T2-weighted image with fat saturation (b). The opposing D11-D12-L1 vertebral bodies (arrows) show changes of the bone marrow in their anterior corners, with T1-hypo-/ hyperintensity (a) and

T2-hyperintensity (b). These findings indicate vascularized fibrous tissue, with edema, and initial fatty marrow replacement in the abovementioned areas (types 1-2 according to Modic). The pathogenesis of these lesions is not infectious but mechanical. The edematous pattern indicates a sort of "degenerative spondylitis"
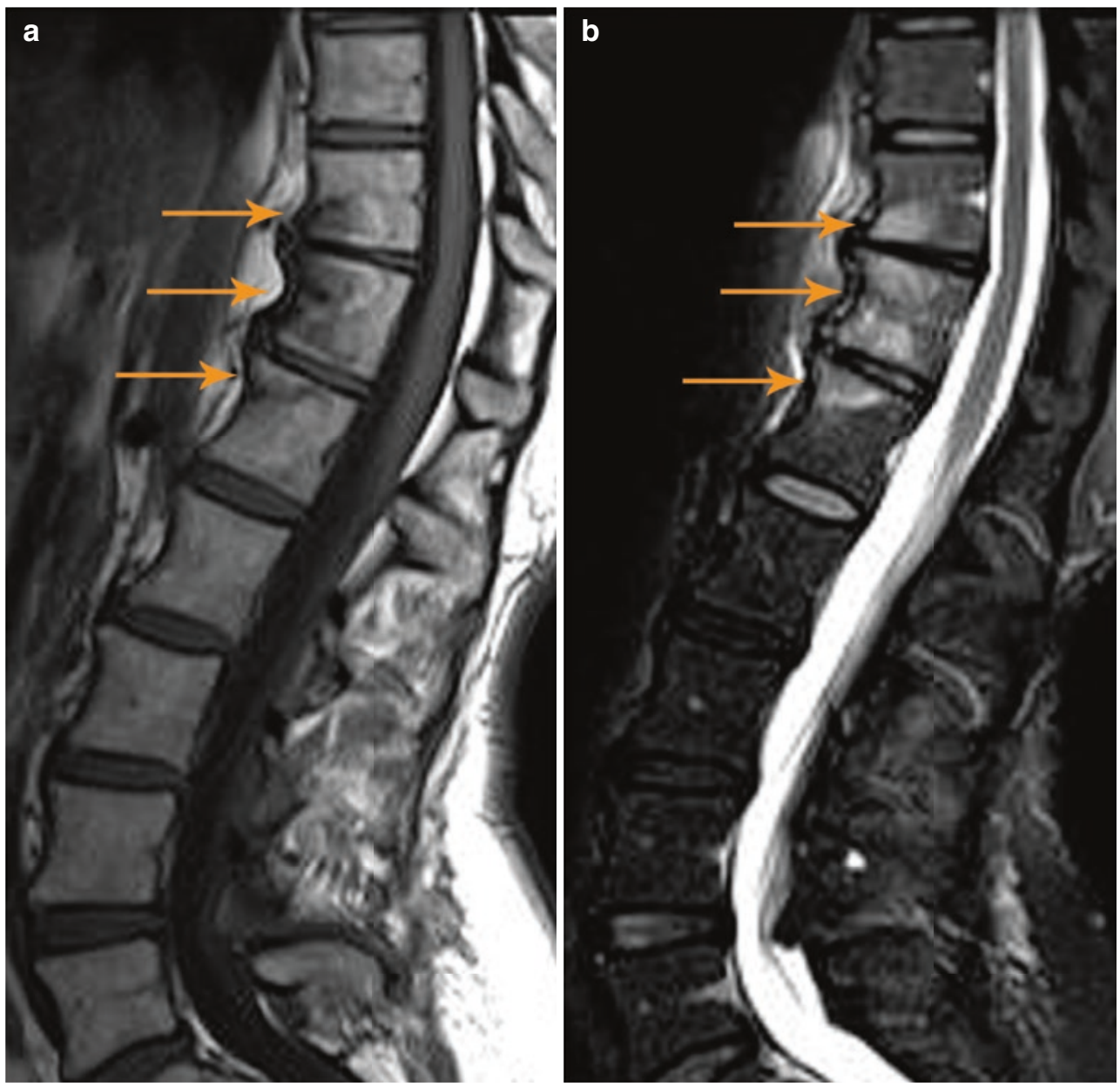